\title{
Impact of heavy sterile neutrinos on the triple Higgs coupling
}

\section{Julien Baglio}

Institute for Theoretical Physics, University of Tübingen, Auf der Morgenstelle 14,

72076 Tübingen, Germany

Institute for Advanced Study, Durham University, Cosin's Hall, Palace Green, Durham DH1

3RL, United Kingdom

Institute for Particle Physics Phenomenology, Department of Physics, Durham University, South

Road, Durham DH1 3LE, United Kingdom

E-mail: julien.baglio@uni-tuebingen.de

\section{Cédric Weiland*}

Institute for Particle Physics Phenomenology, Department of Physics, Durham University, South Road, Durham DH1 3LE, United Kingdom

E-mail: cedric.weiland@durham.ac.uk

\begin{abstract}
New physics beyond the Standard Model is required to give mass to the light neutrinos. One of the simplest ideas is to introduce new heavy, gauge singlet fermions that play the role of right-handed neutrinos in a seesaw mechanism. They could have large Yukawa couplings to the Higgs boson, affecting the Higgs couplings and in particular the triple Higgs coupling $\lambda_{H H H}$, the measure of which is one of the major goals of the LHC and of future colliders. We present a study of the impact of these heavy neutrinos on $\lambda_{H H H}$ at the one-loop level, first in a simplified $3+1$ model with one heavy Dirac neutrino and then in the inverse seesaw model. Taking into account all possible experimental constraints, we find that sizeable deviations of the order of $35 \%$ are possible, large enough to be detected at future colliders, making the triple Higgs coupling a new, viable observable to constrain neutrino mass models. The effects are generic and are expected in any new physics model including TeV-scale fermions with large Yukawa couplings to the Higgs boson, such as those using the neutrino portal.
\end{abstract}

The European Physical Society Conference on High Energy Physics

5-12 July, 2017

Venice

${ }^{*}$ Speaker. 


\section{Introduction}

The Super-Kamiokande experiment firmly established in 1998 that neutrinos oscillate [1], which calls for an extension of the Standard Model (SM) that generates neutrino masses and mixing. One of the simplest possibilities to explain neutrino masses is to add new fermionic gauge singlets that play the role of right-handed neutrinos. These new fermionic states could have large Yukawa couplings to the Higgs boson, having a sizeable impact on the Higgs couplings and opening new search strategies.

The Higgs self-couplings, and in particular the triple Higgs coupling $\lambda_{H H H}$, play a central role in probing electroweak symmetry breaking (EWSB) induced by the Higgs mechanism [2-6]. The measure of $\lambda_{H H H}$ is one of the major goals of the LHC and of the future planned colliders such as the electron-positron International Linear Collider (ILC) or the Future Circular Collider in hadron mode (FCC-hh), a potential $100 \mathrm{TeV}$ follow-up of the LHC. Investigating possible beyond-the-SM (BSM) effects on this coupling is thus very much needed and the effects induced by the heavy neutrinos present in seesaw mechanisms have been overlooked so far.

We present a study of the impact of these heavy neutrinos on $\lambda_{H H H}$, first by considering a simplified 3+1 model where the SM is minimally modified to account for three light massive Dirac neutrinos and one heavy sterile Dirac neutrino; then by considering the inverse seesaw mechanism [7-9] which is a realistic, renormalisable mass model with 9 Majorana neutrinos. Taking into account all theoretical and experimental constraints, we find in both studies [10,11] sizeable effects, of the order of $35 \%$ for large off-shell Higgs momentum $q_{H}^{*}$ and of the order of $10 \%$ for $q_{H}^{*}=500 \mathrm{GeV}$. This is clearly detectable at the FCC-hh and may be probed at the ILC, making the triple Higgs coupling $\lambda_{H H H}$ a new, viable observable for the neutrino sector in order to constraint mass models.

\section{The triple Higgs coupling}

The Higgs field $\Phi$ of the SM can be written as

$$
\Phi=\frac{1}{\sqrt{2}}\left(\begin{array}{c}
\sqrt{2} G^{+} \\
\mathrm{v}+H+\imath G^{0}
\end{array}\right),
$$

where $H$ is the Higgs boson, $G^{0}$ is the neutral Goldstone boson, $G^{ \pm}$are the charged Goldstone bosons, and $\mathrm{v} \simeq 246 \mathrm{GeV}$ is the Higgs vacuum expectation value. After EWSB, the scalar potential of the SM contains the following terms involving the Higgs boson $H$,

$$
V(H)=\frac{1}{2} M_{H}^{2} H^{2}+\frac{1}{3 !} \lambda_{H H H} H^{3}+\frac{1}{4 !} \lambda_{H H H H} H^{4},
$$

where $M_{H}$ is the Higgs boson mass and the tree-level values for the triple and quartic Higgs couplings are $\lambda_{H H H}^{0}=-3 M_{H}^{2} / \mathrm{v}$ and $\lambda_{H H H H}^{0}=-3 M_{H}^{2} / \mathrm{v}^{2}$ respectively.

Our one-loop calculation is performed in the on-shell renormalisation scheme. Our Higgs and electroweak inputs are the Higgs mass $M_{H}$, the $\mathrm{W}$ and $\mathrm{Z}$ boson masses $M_{W}$ and $M_{Z}$, and the electric charge $e$. Details of the calculation and analytical formulas can be found in our articles $[10,11]$. 
Our results will be presented in terms of deviations with respect to the tree-level value $\lambda_{H H H}^{0}$ and to the renormalised one-loop value in the SM $\lambda_{H H H}^{1 r, S M}$ of the triple Higgs coupling,

$$
\begin{aligned}
\Delta^{(1)} \lambda_{H H H} & =\frac{1}{\lambda^{0}}\left(\lambda_{H H H}^{1 r, \text { full }}-\lambda^{0}\right), \\
\Delta^{\mathrm{BSM}} & =\frac{1}{\lambda_{H H H}^{1 r, \mathrm{SM}}}\left(\lambda_{H H H}^{1 r \text { full }}-\lambda_{H H H}^{1 r, \mathrm{SM}}\right) .
\end{aligned}
$$

with $\lambda_{H H H}^{1 \text { rfull }}$ being the one-loop renormalised triple Higgs coupling in the model considered. We will compare our results with the experimental sensitivities to the SM triple Higgs coupling at the LHC, the ILC and the FCC-hh. We use a sensitivity of $\sim 35 \%$ at the high-luminosity run of the LHC (HL-LHC) according to Ref. [12] (see also Ref. [13]), with a scaling of $1 / \sqrt{2}$ to combine ATLAS and CMS results. Using Ref. [14] and again a rescaling we take a sensitivity of $\sim 5 \%$ at the FCC-hh with $3 \mathrm{ab}^{-1}$, and finally we take a sensitivity of $10 \%$ [15] at the $1 \mathrm{TeV}$ ILC with $5 \mathrm{ab}^{-1}$.

\section{Simplified 3+1 model}

In a first study [10], we considered a simplified model that includes 3 light neutrinos and an extra heavy neutrino. All of them are Dirac fermions and the heavy neutrino couples to the SM particles through its mixing with SM fields. This leads to the following couplings between neutrinos and SM bosons, defined in the mass basis,

$$
\begin{aligned}
\mathscr{L} \ni & -\left(\frac{g_{2}}{\sqrt{2}} \bar{\ell}_{i} W^{-} B_{i j} P_{L} n_{j}+\frac{g_{2}}{\sqrt{2} M_{W}} \bar{\ell}_{i} G^{-} B_{i j}\left(m_{\ell_{i}} P_{L}-m_{n_{j}} P_{R}\right) n_{j}\right)+\text { H.c. } \\
& -\frac{g_{2}}{2 \cos \theta_{W}} \bar{n}_{i} \not\left(B^{\dagger} B\right)_{i j} P_{L} n_{j}+\frac{l g_{2}}{2 M_{W}} \bar{n}_{i}\left(B^{\dagger} B\right)_{i j} G^{0}\left(-m_{n_{i}} P_{L}+m_{n_{j}} P_{R}\right) n_{j} \\
& -\frac{g_{2}}{2 M_{W}} \bar{n}_{i}\left(B^{\dagger} B\right)_{i j} H\left(m_{n_{i}} P_{L}+m_{n_{j}} P_{R}\right) n_{j},
\end{aligned}
$$

where $\ell_{i}$ are the charged leptons, $n_{i}$ are the Dirac neutrinos of mass $m_{1 \ldots 4}, g_{2}$ is the $\mathrm{SU}(2)$ coupling constant, and $B$ is a $3 \times 4$ mixing matrix.

The most relevant experimental constraints on our model come from electroweak precision observables (EWPO) and in particular from the global fit performed in [16, 17]. We have also taken into account constraints on the mixing matrix $B$ coming from neutrino oscillations [18] with $\delta_{C P}=0$. We also require two theoretical constraints. The loop expansion has to remain perturbative, and we apply either a loose (tight) bound of

$$
\left(\frac{\max \left|C_{i 4}\right| g_{2} m_{n_{4}}}{2 M_{W}}\right)^{3}<16 \pi(2 \pi) .
$$

Since for fixed mixing the heavy neutrino width grows with its mass, we require as well $\Gamma_{n_{4}} \leq 0.6 m_{n_{4}}$ for the quantum state to be a definite particle.

For our numerical study, SM parameter values were taken from the Particle Data Group [19] (with the exception of the SM Higgs boson mass fixed to $M_{H}=125 \mathrm{GeV}$ ). Taking $B_{\tau 4}=0.087$, $B_{e 4}=B_{\mu 4}=0$, Fig. 1 displays the one-loop induced deviation of $\lambda_{H H H}$ from its tree-level value while the insert presents the size of the corrections coming from the heavy neutrino. With these 


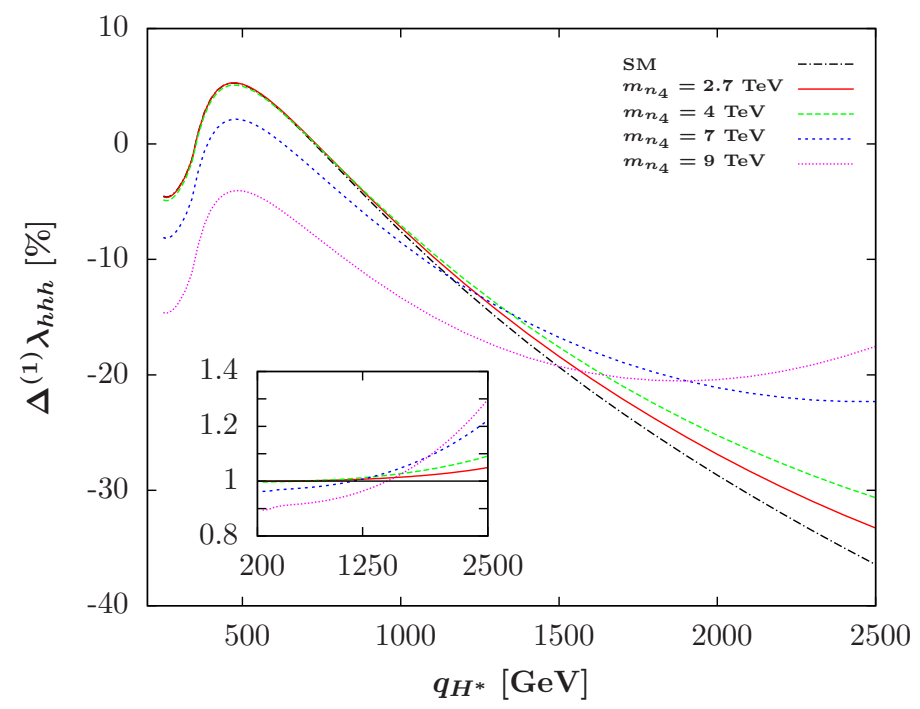

Figure 1: One-loop corrections to the triple Higgs coupling $\lambda_{H H H}$ (in \%) as a function of the momentum $q_{H^{*}}$ of the splitting $H^{*}\left(q_{H^{*}}\right) \rightarrow H H$ (in GeV). The ratio of the genuine BSM contribution to $\lambda_{H H H}$ with respect to the one-loop SM contribution is shown in the insert.

mixing parameters, a heavy neutrino mass of $m_{n_{4}}=2.7 \mathrm{TeV}$ corresponds to an effective coupling to the Higgs equal to the one of the top quark while $m_{n_{4}}=7 \mathrm{TeV}$ leads to the saturation of the tight perturbativity bound and $m_{n_{4}}=9 \mathrm{TeV}$ saturates the width constraint. We observe that in the SM, the largest positive correction is at $q_{H^{*}} \simeq 500 \mathrm{GeV}$, where the BSM contribution decreases it to $-9 \%$ at $m_{n_{4}}=9 \mathrm{TeV}$. The largest negative correction comes at larger momentum where the deviation from the SM increases with larger $m_{n_{4}}$, reaching $+30 \%$ for $m_{n_{4}}=9 \mathrm{TeV}$ at $q_{H^{*}}=2500 \mathrm{GeV}$.

This behaviour leads us to chose $q_{H^{*}} \simeq 500 / 2500 \mathrm{GeV}$ as two most interesting off-shell momenta and to study the size of the BSM corrections induced by the heavy neutrino as a function of its mass and couplings. This is presented in Fig. 2 for $q_{H^{*}}=500 \mathrm{GeV}$ (left) and $q_{H^{*}}=2.5 \mathrm{TeV}$ (right). The largest effects are present in the high mixing / high heavy neutrino mass region, reaching slightly less that $10 \%$ negative deviation at $q_{H^{*}}=500 \mathrm{GeV}$ (less that $-5 \%$ with the tight perturbative bound displayed in red) and around $+30 \%$ increase at $q_{H^{*}}=2.5 \mathrm{TeV}$ (slightly less that $+25 \%$ with the tight perturbative bound). This is always below the HL-LHC sensitivity (35\%), but clearly visible at the FCC-hh (5\%) and potentially visible at the ILC (10\%).

\section{Inverse seesaw model}

In order to confirm the results obtained in the simplified $3+1$ model, we performed in our next study [11] the analysis of the one-loop corrections to $\lambda_{H H H}$ in a renormalisable, low-scale seesaw model, namely the inverse seesaw (ISS) [7-9]. We add to the SM Lagrangian 6 fermionic gauge singlets, 3 states with positive lepton number $L=+1\left(v_{R}\right)$ and 3 states with negative lepton number $L=-1(X)$ with the following Yukawa couplings and mass terms,

$$
\mathscr{L}_{\mathrm{ISS}}=-Y_{v}^{i j} \overline{\ell_{i}} \widetilde{\Phi} v_{R j}-M_{R}^{i j} \overline{v_{R i}^{C}} X_{j}-\frac{1}{2} \mu_{X}^{i j} \overline{X_{i}^{C}} X_{j}+\text { H.c. }
$$



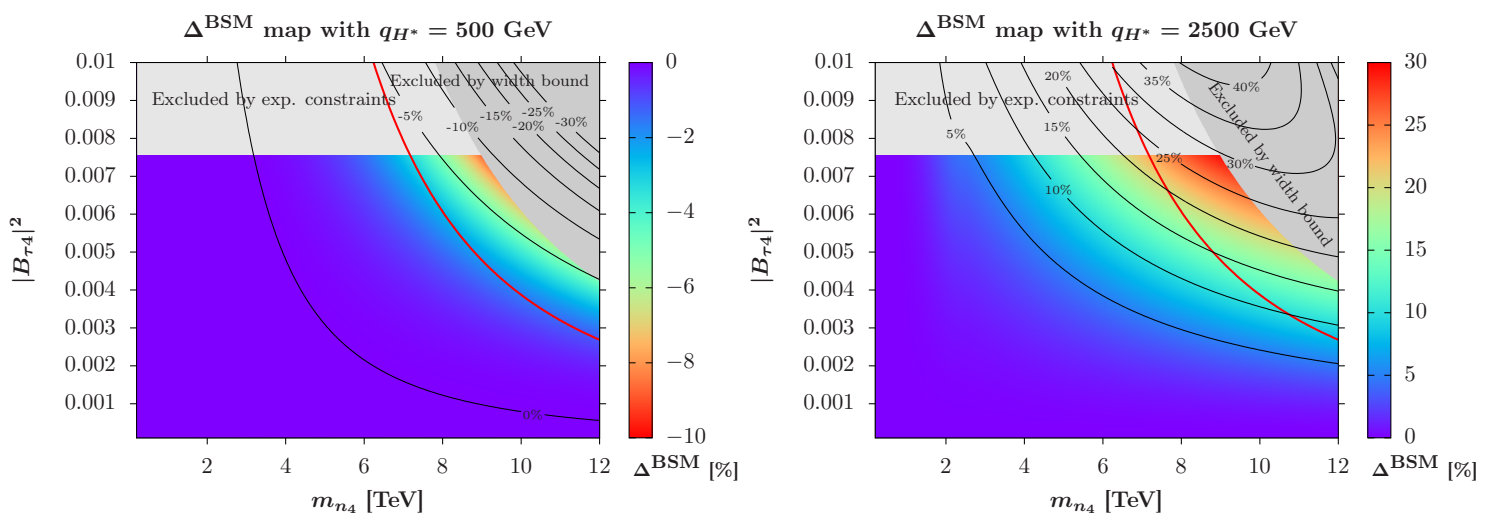

Figure 2: Contour maps of the neutrino corrections $\Delta^{\mathrm{BSM}}$ to the triple Higgs coupling $\lambda_{H H H}$ (in $\%$ ) as a function of the two neutrino parameters $\left|B_{\tau 4}\right|^{2}$ and $m_{n_{4}}$ (in TeV), at a fixed off-shell Higgs momentum $q_{H^{*}}=500 \mathrm{GeV}$ (left) and $q_{H^{*}}=2500 \mathrm{GeV}$ (right). The other heavy neutrino mixing parameters are set to zero. The light grey area is excluded by the experimental constraints and the darker grey area is excluded from having $\Gamma_{n_{4}}>0.6 m_{n_{4}}$ while the red line corresponds to the tight perturbativity bound.

leading after EWSB to 9 Majorana neutrinos $N_{i}$. Thanks to the two scale parameters $\mu_{X}$ and $M_{R}$ it is possible to decouple the neutrino mass generation from the mixing between the active neutrinos and the fermionic gauge singlets. The light neutrino masses are suppressed by the small leptonnumber breaking parameter $\mu_{X}$. It is then possible to have at the same time Yukawa couplings $Y_{v}$ of order 1 and $M_{R} \sim 1 \mathrm{TeV}$, which is within reach of the LHC and low energy experiments.

The calculation of the one-loop corrections to $\lambda_{H H H}$ in the ISS is very similar to the calculation in the 3+1 model, but with Majorana neutrinos instead of Dirac neutrinos. All formulae are available in the appendix B of our article [11]. The set of constraints changes, though. The constraints from low-energy neutrino data are implemented via the $\mu_{X}$-parametrisation [20],

$$
\mu_{X}=M_{R}^{T} m_{D}^{-1} U_{\mathrm{PMNS}}^{*} m_{v} U_{\mathrm{PMNS}}^{\dagger} m_{D}^{T-1} M_{R},
$$

at the lowest order in the seesaw expansion parameter $m_{D} M_{R}^{-1}$. Terms beyond this order are also included in our analysis and are given in the appendix A of our article [11]. Charged lepton flavour violation bounds are taken into account (see e.g. Ref. [21]) as well as the global fit to EWPO and the lepton universality tests [22]. Theoretical constraint on the heavy neutrino widths $\Gamma_{N_{i}} \leq 0.6 m_{N_{i}}$ and the Yukawa perturbativity constraint $\left|Y_{v}^{2}\right|<1.5 \times 4 \pi$ are also included.

Representative numerical results of our study of the size of the BSM corrections induced by the heavy neutrinos are displayed in Fig. 3. We have used a diagonal Yukawa texture $Y_{v}=\left|Y_{v}\right| I_{3}$ and a hierarchical heavy neutrino mass matrix with $M_{R}=\operatorname{diag}\left(1.51 M_{R}, 3.59 M_{R}, M_{R}\right)$, where $M_{R}$ corresponds to the seesaw scale. This choice of textures for the two matrices leads to the largest effects in the ISS, reaching the $+30 \%$ deviation for a large momentum $q_{H^{*}}=2.5 \mathrm{TeV}$ and the $-8 \%$ deviation for $q_{H^{*}}=500 \mathrm{GeV}$, for $M_{R}$ around $9 \mathrm{TeV}$ and large Yukawa couplings. These results can be approximated by the following formula that is used for the green lines in Fig. 3 ,

$$
\Delta_{\text {approx }}^{\mathrm{BSM}}=0.51 \frac{(1 \mathrm{TeV})^{2}}{M_{R}^{2}}\left(8.45 \operatorname{Tr}\left(Y_{v} Y_{v}^{\dagger} Y_{v} Y_{v}^{\dagger}\right)-0.145 \operatorname{Tr}\left(Y_{v} Y_{v}^{\dagger} Y_{v} Y_{v}^{\dagger} Y_{v} Y_{v}^{\dagger}\right)\right) .
$$



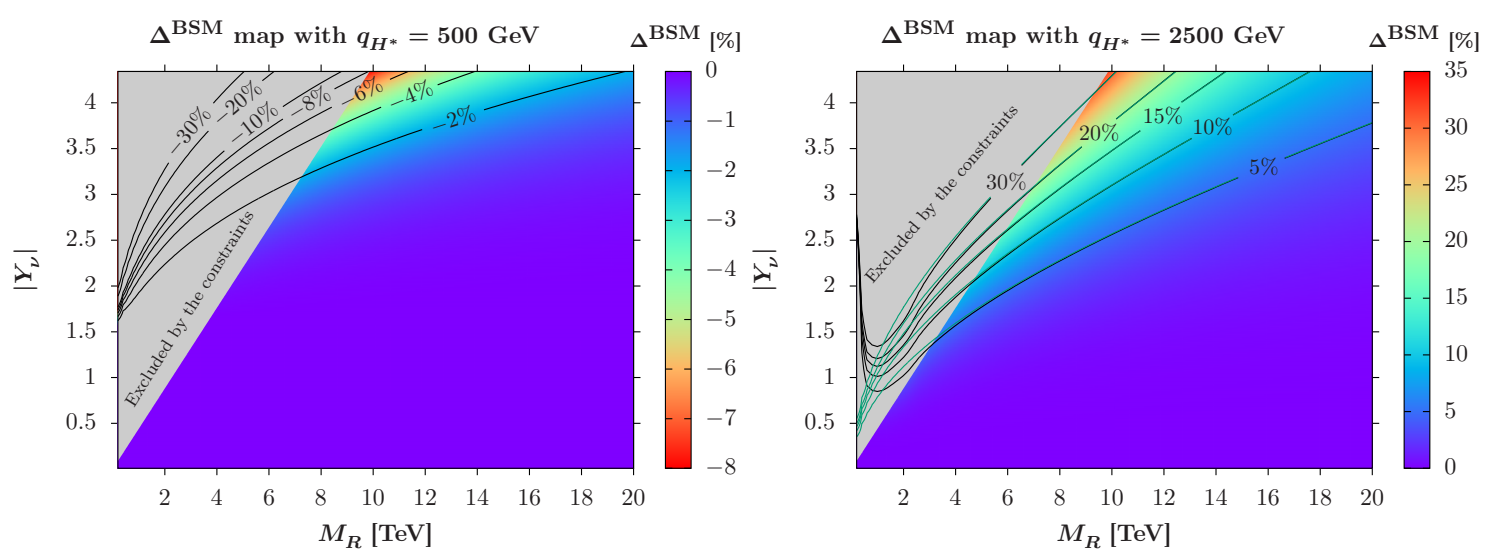

Figure 3: Contour maps of the heavy neutrino correction $\Delta^{\mathrm{BSM}}$ to the triple Higgs coupling $\lambda_{H H H}$ (in $\%$ ) as a function of the neutrino parameters $M_{R}$ (in TeV) and $\left|Y_{V}\right|$ in the $\mu_{X}$-parametrisation, at a fixed off-shell Higgs momentum $q_{H^{*}}=500 \mathrm{GeV}$ and $q_{H^{*}}=2.5 \mathrm{TeV}$ (right). We have used a diagonal Yukawa texture $Y_{v}$ with parameter $\left|Y_{v}\right|$ and a hierarchical heavy neutrino mass matrix, varying the seesaw scale $M_{R}$. The grey area is excluded by the constraints on the model and the green lines on the right figure are the approximated contour lines using eq.(4.3), while the black lines correspond to the full calculation.

The larger number of heavy neutrinos compared to the simplified 3+1 model would naively induce larger corrections in the ISS. However, the experimental constraints are stronger in this case, which leads to results similar to the simplified $3+1$ analysis of the previous section. This confirms our previous conclusion that the triple Higgs coupling $\lambda_{H H H}$ is a new, viable observable to constrain neutrino mass models.

\section{Conclusion}

The indubitable observation of neutrino oscillations requires the addition of BSM physics to generate neutrino masses and mixing and one of the simplest and well-motivated ideas adds new right-handed sterile neutrinos to the SM, leading to seesaw models. We have introduced the triple Higgs coupling as a new observable to constraint these neutrino mass models and we have found, first in a simplified 3+1 model and then in the inverse seesaw, that the one-loop effects induced by these heavy neutrinos can be as large as $30 \%$ with respect to the SM one-loop prediction. This is measurable at future colliders and it provides a new, complementary probe in the $\mathscr{O}(10) \mathrm{TeV}$ range of the heavy neutrino masses. We stress that these effects are generic and would be expected in any model containing $\mathrm{TeV}$ scale fermions with large Higgs couplings, such as those using the neutrino portal.

\section{Acknowledgments}

J. B. acknowledges the support from the Institutional Strategy of the University of Tübingen (DFG, ZUK 63), from the DFG Grant JA 1954/1., from the Kepler Center of the University of Tübingen, as well as from his Durham Senior Research Fellowship COFUNDed between Durham University and the European Union under grant agreement number 609412. C. W acknowledges 
the support from the European Research Council under the European Union's Seventh Framework Programme (FP/2007-2013) / ERC Grant NuMass agreement n. [617143] and partial support from the European Union's Horizon 2020 research and innovation programme under the Marie Skłodowska-Curie grant agreements No. 690575 and No. 674896.

\section{References}

[1] Super-Kamiokande collaboration, Y. Fukuda et al., Phys. Rev. Lett. 81 (1998) 1562-1567.

[2] P. W. Higgs, Phys. Lett. 12 (1964) 132.

[3] F. Englert and R. Brout, Phys. Rev. Lett. 13 (1964) 321.

[4] P. W. Higgs, Phys. Rev. Lett. 13 (1964) 508.

[5] G. S. Guralnik, C. R. Hagen and T. W. B. Kibble, Phys. Rev. Lett. 13 (1964) 585.

[6] P. W. Higgs, Phys. Rev. 145 (1966) 1156.

[7] R. N. Mohapatra and J. W. F. Valle, Phys. Rev. D 34 (1986) 1642.

[8] R. N. Mohapatra, Phys. Rev. Lett. 56 (1986) 561.

[9] J. Bernabeu, A. Santamaria, J. Vidal, A. Mendez and J. W. F. Valle, Phys. Lett. B 187 (1987) 303.

[10] J. Baglio and C. Weiland, Phys. Rev. D 94 (2016) 013002.

[11] J. Baglio and C. Weiland, JHEP 1704 (2017) 038.

[12] CMS Collaboration [CMS Collaboration], CMS-PAS-FTR-15-002.

[13] P. Campana, M. Klute and P. Wells, Ann. Rev. Nucl. Part. Sci. 66 (2016) 273.

[14] H. J. He, J. Ren and W. Yao, Phys. Rev. D 93 (2016) 015003.

[15] K. Fujii et al., arXiv:1506.05992 [hep-ex].

[16] F. del Aguila, J. de Blas and M. Perez-Victoria, Phys. Rev. D 78 (2008) 013010.

[17] J. de Blas, EPJ Web Conf. 60 (2013) 19008.

[18] M. C. Gonzalez-Garcia, M. Maltoni and T. Schwetz, JHEP 1411 (2014) 052.

[19] C. Patrignani et al. [Particle Data Group], Chin. Phys. C 40 (2016) 100001.

[20] E. Arganda, M. J. Herrero, X. Marcano and C. Weiland, Phys. Rev. D 91 (2015) 015001.

[21] A. M. Baldini et al. [MEG Collaboration], Eur. Phys. J. C 76 (2016) no.8, 434.

[22] E. Fernandez-Martinez, J. Hernandez-Garcia and J. Lopez-Pavon, JHEP 1608 (2016) 033. 